\title{
Diferencias según género en el tiempo empleado por adolescentes en actividad sedentaria y actividad física en diferentes segmentos horarios del día Gender differences in time spent by adolescents in sedentary and physical activity in different day segments \\ Vicente Javier Beltrán Carrillo, Ana Cristina Sierra,Alejandro Jiménez Loaisa, David González-Cutre, Celestina Martínez Galindo, Eduardo Cervelló \\ Universidad Miguel Hernández de Elche
}

\begin{abstract}
Resumen. El objetivo de este estudio fue analizar si existían diferencias según género en el tiempo empleado por los adolescentes en actividad sedentaria y actividad física ligera, moderada y vigorosa en diferentes segmentos horarios del día. Estas diferencias se analizaron tanto entre semana como en fin de semana. La muestra inicial de este estudio se compuso de 206 estudiantes de $3^{\circ}$ y $4^{\circ}$ de Educación Secundaria Obligatoria ( $M=15,07$; $D T=0,86)$. Las mediciones de la actividad sedentaria y la actividad física se realizaron con acelerómetros ActiGraph GT3X que los adolescentes llevaron durante una semana. Las chicas mostraron mayor actividad sedentaria que los chicos en el segmento horario 'Precolegio' (de 06:30 a 08:30 horas), mientras que los chicos presentaron mayor actividad física vigorosa en este segmento horario. Los chicos mostraron mayor actividad física vigorosa en los segmentos horarios ‘Colegio' (de 08:31 a 14:30 horas), 'Comida entre semana' (de 14:31 a 16:00 horas), 'Tarde 1 entre semana' (de 16:01 a 18:30 horas) y 'Tarde 2 entre semana' (de 18:31 a 21:00 horas). Las chicas presentaron mayor actividad sedentaria en el segmento 'Tarde 2 entre semana'. Durante el fin de semana, los chicos mostraron mayor actividad vigorosa en el segmento horario 'Tarde 1' (de 16:01 a 18:30 horas). Junto a la discusión de los resultados, este artículo plantea una serie de recomendaciones para la promoción de la actividad física en las chicas. Palabras clave. Actividad física, adolescentes, género.
\end{abstract}

\begin{abstract}
The aim of this study was to analyse the existence of possible gender differences in time spent by adolescents in sedentary activity and light, moderate and vigorous physical activity in different day segments. These differences were analysed both on weekdays and weekend days. The initial sample of this study consisted of 206 secondary school students $(M=15.07 ; S D=0.86)$. Sedentary and physical activity were measured with ActiGraph GT3X accelerometers, which were worn by the adolescents during one week. Girls showed higher levels of sedentary activity during 'Pre-school time' (from 06:30 to 08:30 hours), whereas boys showed more vigorous physical activity during this segment of the day. Boys showed higher levels of vigorous physical activity during 'School' (from 08:31 to 14:30 hours), 'Weekday lunches' (from 14:31 to 16:00 hours), 'Weekday afternoons' (from 16:01 to 18:30 hours), and 'Weekday evenings' (from 18:31 to 21:00 hours). Girls showed more sedentary activity during 'Weekday evenings'. Finally, boys showed higher levels of vigorous physical activity during 'Weekend afternoons' (from 16:01 to 18:30 hours). Besides the discussion of the results, this article proposes several recommendations to promote girls' participation in physical activity.
\end{abstract}

Keywords. Physical activity, adolescents, gender.

\section{Introducción}

La inactividad física de la población está considerada en la actualidad uno de los factores de riesgo más importantes de las enfermedades no transmisibles, como pueden ser la obesidad, la diabetes tipo II, o las enfermedades cardiovasculares (World Health Organization, 2011). Los insuficientes niveles de actividad física (AF) preocupan especialmente en niños y adolescentes, ya que este sector de población se encuentra en una etapa clave para la adquisición y consolidación de hábitos saludables (Telama et al., 2014; Welk, Eisenmann, y Dollman, 2006). Sin embargo, diversas investigaciones muestran que un alto porcentaje de niños y adolescentes no practica suficiente AF de forma habitual. Concretamente, un estudio en el que participaron 72.845 adolescentes de 34 países de diferentes partes del mundo concluyó que solamente el 23,8\% de los chicos y el 15,4\% de las chicas cumplían con las recomendaciones mínimas de 60 minutos diarios de AF moderada-vigorosa al menos 5 días a la semana (Guthold, Cowan, Autenrieth, Kann, y Riley, 2010). Este mismo estudio indicó que en más de la mitad de los países estudiados, más de un tercio de los adolescentes dedicaron 3 horas o más al día a actividades sedentarias, excluyendo las horas de estar sentado en el colegio y de hacer los deberes escolares. En el contexto español, el estudio de Ramos, Rivera, Moreno y Jiménez-Iglesias (2012), llevado a cabo con una muestra representativa de la población adolescente de España compuesta por 21.811 participantes, indicó que sólo el 45,1\% de los chicos y el $30 \%$ de las chicas cumplía con el criterio de realizar AF moderada-vigorosa al menos 60 minutos al día, 5 días a la semana. Además, los adolescentes españoles mostraron una elevada participación en conductas sedentarias, dedicando una media diaria de 2,37 horas a ver la televisión, 1,09 horas a jugar con el ordenador o la consola y 1,11 horas a usar el ordenador para actividades como chatear, navegar por

Fecha recepción: 26-05-15. Fecha de aceptación: 19-04-16 Vicente Beltrán Carrillo vbeltran@umh.es internet, mandar e-mails o hacer los deberes. Este estudio también demostró que los adolescentes con mejor estado de salud fueron aquellos que mostraban alta frecuencia deAF moderada-vigorosa y baja frecuencia de conductas sedentarias.

Las evidencias de la literatura convierten la promoción de la AF en los jóvenes en un objetivo prioritario de salud pública. No obstante, para el diseño y puesta en práctica de programas y estrategias de promoción de la $\mathrm{AF}$ es necesario conocer los patrones de $\mathrm{AF}$ de niños y adolescentes y los factores que influyen en esos patrones (Peiró-Velert, Valenciano-Valcárcel, Beltrán-Carrillo, y Devís-Devís, 2014). Este conocimiento es necesario para realizar intervenciones más adecuadas, que se ajusten a las preferencias y necesidades de esta población.

Respecto a los patrones de AF, es necesario analizar la participación de niños y adolescentes en las diferentes actividades según nivel de intensidad, es decir, tanto en actividad sedentaria, como en AF ligera, moderada y vigorosa (Bailey et al., 2012). De hecho, realizar suficiente AF moderada-vigorosa no garantiza un estilo de vida activo y saludable si las personas mantienen niveles muy elevados de actividad sedentaria y niveles muy bajos de AF ligera (Arto, 2015).

Respecto a los factores que influyen en los patrones de AF, se ha prestado especial atención a la variable género y la mayoría de estudios señalan que los chicos mantienen un estilo de vida más activo que las chicas (Guthold et al., 2010; Laguna et al., 2013; Ramos et al., 2012; Yan, Voorhees, Beck, y Wang, 2014). Sin embargo, poco se sabe aún de los patrones de AF de chicos y chicas en diferentes segmentos horarios del día y de las diferencias que pueden existir según género (Aibar et al., 2014; Bailey et al., 2012). Para este tipo de estudios se aconseja el uso de acelerómetros, que garantizan mediciones más objetivas y precisas que otros métodos de medida de la AF como los instrumentos de autoinforme (Aibar et al., 2014; Bailey et al., 2012). Los principales resultados de estos estudios se detallan a continuación.

Bailey et al. (2012), en su estudio con niños y adolescentes de 10 a 14 años de Reino Unido, encontraron que las chicas mostraban mayor tiempo en AF ligera que los chicos durante el segmento horario correspondiente al transporte al centro escolar. Las chicas mostraban más 
actividad sedentaria y AF ligera que los chicos durante el recreo escolar de la mañana y el descanso para el almuerzo (lunch), mientras que los chicos presentaban mayor AF vigorosa en el recreo de la mañana y más AF moderada y vigorosa en el descanso para el almuerzo (lunch). Durante las horas de clase, las chicas mostraron mayor actividad sedentaria. No existieron diferencias significativas en ninguna variable durante el segmento horario ‘después del colegio’ (desde el final de laúltima clase del colegio hasta las $18.30 \mathrm{~h}$ ).

Aibar et al. (2014), en su estudio con una muestra de adolescentes españoles y franceses, señalaron que los chicos acumulaban más AF moderada-vigorosa que las chicas en todos los segmentos horarios establecidos para los días entre semana ('tiempo de transporte al colegio', 'tiempo en la escuela', 'tiempo fuera de la escuela') y los fines de semana ('mañana', 'tarde', 'noche'). Las mayores diferencias se hallaron en los segmentos horarios 'mañana' del fin de semana, 'tiempo fuera de la escuela' y ‘tiempo en la escuela'.

Pese a que los estudios mencionados sientan las bases de una línea de investigación necesaria para generar un conocimiento que permita mejorar las estrategias de promoción de la AF, aún son muy escasos este tipo de estudios en la literatura. Además, estos estudios dividen el día en pocos segmentos horarios, el estudio de Bailey et al. (2012) sólo analiza segmentos horarios entre semana y el estudio de Aibar et al. (2014) no analiza la actividad sedentaria y laAF ligera. Por lo tanto, el objetivo de este estudio, llevado a cabo con una muestra de adolescentes y basado en mediciones con acelerómetro, fue analizar si existían diferencias según género en el tiempo empleado en actividad sedentaria y AF ligera, moderada y vigorosa en diferentes segmentos horarios del día, tanto entre semana como en fin de semana.

\section{Método}

\section{Participantesymedidas}

La muestra inicial de este estudio se compuso de 206 estudiantes de $3^{\circ}$ y $4^{\circ}$ de Educación Secundaria Obligatoria (118 chicas y 88 chicos), de edades comprendidas entre los 14 y los 17 años $(M=15,07 ; D T=0,86)$. Los participantes procedían de tres centros escolares de la provincia de Alicante. Estos centros eran de titularidad pública y el alumnado procedía de familias cuyo estatus socioeconómico era medio.

Para la medición del tiempo empleado en actividad sedentaria y AF ligera, moderada y vigorosa en cada segmento horario del día se utilizaron acelerómetros ActiGraph GT3X, que los adolescentes llevaron durante una semana. Estos acelerómetros han demostrado ser una herramienta válida y fiable para medir la AF y las conductas sedentarias de los adolescentes (Santos-Lozano et al., 2012). Los datos de aceleración procedentes del eje vertical fueron filtrados por el acelerómetro y almacenados en su memoria en counts (unidad de medida que utiliza el acelerómetro) y en epochs o fragmentos temporales de 60 segundos.

\section{Procedimiento}

Para realizar este estudio se solicitó autorización a la Consejería de Educación de la Comunidad Valenciana, a los centros educativos y a los padres de los alumnos implicados, que firmaron un consentimiento informado. El estudio fue aprobado por el comité ético de investigación de la Universidad Miguel Hernández de Elche y la participación en el mismo fue totalmente voluntaria. Para realizar las medidas, se informó a los alumnos del objetivo y procedimiento del estudio. Se explicó a los estudiantes cómo debían utilizar los acelerómetros durante la semana. Los acelerómetros debían colocarse en el lado derecho, entre la cinturay la cadera, mediante un cinturón elástico, para el registro de su actividad durante siete días consecutivos. Debían ponerse el acelerómetro todos los días cuando se despertaran, quitándoselo solamente para dormir, ducharse, u otras actividades que pudieran dañar el acelerómetro, como por ejemplo la natación. Una semana después, se recogieron los acelerómetros con los datos ya registrados para su posterior análisis.

\section{Reducción de datos}

El volcado de los datos procedentes de los acelerómetros se realizó con la ayuda del software Actilife 6. Los periodos de registro iguales o superiores a 10 minutos consecutivos de 0 counts, incluyendo 1 o 2 minutos consecutivos con valores iguales o inferiores a 100 counts, fueron considerados periodos en los que los participantes no llevaron el acelerómetro y excluidos del análisis (Aibar et al., 2014).

Para el cálculo del tiempo (minutos) en actividad sedentaria y en las diferentes intensidades de AF se tuvieron en cuenta los puntos de corte establecidos para jóvenes por Evenson, Cattellier, Gill, Ondrak, y McMurray (2008), recomendados también por Trost, Loprinzi, Moore, y Pfeifer (2011): sedentaria 0-100 counts por minuto (<1,5 METs), ligera 101-2295 counts por minuto $(\geq 1,5 \mathrm{y}<4$ METs), moderada 2296-4011 counts por minuto ( $\geq 4 \mathrm{y}<6$ METs), y vigorosa $\geq 4012$ counts por minuto ( $\geq 6 \mathrm{METs})$.

Los segmentos horarios establecidos para la división de un día entre semana fueron: 'Precolegio' (06:30 a 08:30 h), ‘Colegio' (08:31 a 14:30 h), 'Comida' (14:31 a 16:00 h), 'Tarde 1' (16:01 a 18:30 h), 'Tarde 2' (18:31 a 21:00 h) y ‘Noche’ (21:01 a 23:59 h). Los segmentos horarios establecidos para la división de un día en fin de semana fueron: 'Mañana' (06:30 a 14:30 h), 'Comida' (14:31 a 16:00 h), ‘Tarde 1' (16:01 a 18:30 h), ‘Tarde 2' (18:31 a 21:00 h), 'Noche’ (21:01 a 23:59 h), 'Madrugada sábado’ y ‘Madrugada domingo' (ambos de 00:00 a 06:29 h). Estos segmentos horarios fueron decididos por el grupo de investigación, tras un proceso de discusión y llegado a un consenso. Se intentó diferenciar entre diversos momentos del día que resultaban de interés para el objetivo del estudio. Se diferenció entre 'Tarde 1' y 'Tarde 2' porque la toma de datos se realizó durante los meses de noviembre y diciembre. Durante estos meses en la provincia de Alicante la puesta de sol es cercana a las $18 \mathrm{~h}$ y podemos entender que a partir de las $18: 30 \mathrm{~h}$ ha anochecido claramente. Así pues, se dividió la tarde en dos segmentos horarios, uno en el que predominantemente era de día y otro en el que ya había caído la noche.

Para que los datos de los participantes fueran incluidos en el análisis final se establecieron dos criterios de inclusión. En primer lugar, el tiempo de medición debía de ser al menos del 80\% del tiempo estándar en cada segmento horario, entendiendo como tiempo estándar aquel en el que al menos el 70\% de los participantes había llevado el acelerómetro (Aibar et al., 2014; Catellier et al., 2005). En segundo lugar, los participantes debían mostrar un tiempo de medición válido en al menos tres días para los segmentos horarios entre semana y al menos un día para los segmentos horarios del fin de semana (Aibar et al., 2014). Tras la aplicación de estos criterios de inclusión, la muestra inicial del estudio se vio reducida en los diferentes segmentos horarios. La muestra final para cada segmento horario figura en las tablas 2 y 3.

\section{Análisis estadístico}

Se calculó la media $(M)$ y la desviación típica $(D T)$ del tiempo dedicado a actividad sedentaria y AF ligera, moderada y vigorosa en todos los segmentos horarios (datos expresados en minutos/día). Para analizar si existían diferencias en actividad sedentaria y AF ligera, moderada y vigorosa según género, se calcularon MANCOVAs para cada segmento horario. Para controlar un posible sesgo, debido a las variaciones en el tiempo en que cada adolescente llevó el acelerómetro, el tiempo total de medición en cada segmento horario fue incluido como covariable en los análisis. Asimismo, se realizaron ANCOVAS de continuación para analizar concretamente en qué variables dependientes existían diferencias según género (tiempo total medido en cada segmento horario como covariable). Estos análisis se realizaron con el software SPSS 21.

\section{Resultados}

Los resultados mostraron diferencias estadísticamente significativas $(p<.05)$ según género en diferentes segmentos horarios del día entre semana (Tabla 1). En la Tabla 2, se indica la My la DT de los minutos en actividad sedentaria y en las diferentes intensidades de práctica de AF entre semana. En este apartado de resultados, estos datos se expresan como $M \pm D T$. En el segmento horario 'Precolegio' las chicas mostraron mayor actividad sedentaria que los chicos (25,51 $\pm 10,15$ vs. $23,51 \pm$ 10,30; $p<.05, \eta^{2}=.025$ ), mientras que los chicos mostraron mayor 
Tabla 1.

Resultados de los MANCOVAS y ANCOVAS realizados para analizar las diferencias en en actividad sedentaria y en actividad física según género.

\begin{tabular}{|c|c|c|c|c|c|c|c|c|c|c|c|}
\hline \multirow{3}{*}{$\begin{array}{c}\text { Segmentos } \\
\text { horarios }\end{array}$} & \multirow{2}{*}{\multicolumn{3}{|c|}{ MANCOVAS }} & \multirow{2}{*}{\multicolumn{8}{|c|}{$\begin{array}{c}\text { ANCOVAS } \\
\text { Ligera Actividac }\end{array}$}} \\
\hline & & & & & & & & & & & \\
\hline & $\begin{array}{l}\text { Lambda } \\
\text { de Wilks }\end{array}$ & $F$ & $\eta^{2}$ & $F$ & $\eta^{2}$ & $F$ & $\eta^{2}$ & $F$ & $\eta^{2}$ & $F$ & $\eta^{2}$ \\
\hline \multicolumn{12}{|l|}{ Entre semana } \\
\hline Precolegio & .941 & $2,463^{*}$ & .059 & $4,057^{*}$ & .025 & .053 & .003 & .147 & .001 & $6,155^{*}$ & .037 \\
\hline Colegio & .870 & $6,548^{* * * *}$ & .130 & .009 & .000 & 3,378 & .019 & 2,234 & .012 & $21,891 * * *$ & .110 \\
\hline Comida & .943 & $2,571^{*}$ & .057 & .197 & .001 & .719 & .004 & 1,332 & .008 & $4,230^{*}$ & .024 \\
\hline Tarde 1 & .903 & $4,453^{* *}$ & .097 & 2,711 & .016 & .164 & .001 & .020 & .000 & $13,972 * * *$ & 077 \\
\hline Tarde 2 & .882 & $5,368 * * *$ & .118 & $8,238 * *$ & .048 & 2,449 & .015 & .054 & .000 & $16,250 * * *$ & 091 \\
\hline Noche & .956 & 1,693 & .044 & 2,181 & .014 & .300 & .002 & 2,554 & .017 & 3,289 & .022 \\
\hline \multicolumn{12}{|l|}{ Fin de semana } \\
\hline Mañana & .979 & .878 & .021 & .765 & .005 & .011 & .000 & .778 & .005 & 3,286 & .019 \\
\hline Comida & .973 & 1,149 & .027 & 3,316 & .019 & 2,137 & .012 & .029 & .000 & 1,107 & .006 \\
\hline Tarde 1 & .925 & 3,363* & .075 & 2,225 & .013 & .000 & .000 & .720 & .004 & 7,733** & .044 \\
\hline Tarde 2 & .988 & .509 & .012 & .007 & .000 & .004 & .000 & .167 & .001 & .710 & .004 \\
\hline Noche & .936 & 2,894 & .064 & 2,009 & .012 & .255 & .001 & 3,569 & .020 & 1,146 & .007 \\
\hline $\begin{array}{l}\text { Madrugada } \\
\text { sábado }\end{array}$ & .980 & .399 & .020 & .105 & .002 & .115 & .002 & .067 & .001 & .831 & .014 \\
\hline $\begin{array}{l}\text { Madrugada } \\
\text { domingo }\end{array}$ & .983 & .485 & .017 & .312 & .004 & .085 & .001 & .168 & .002 & 1,431 & 016 \\
\hline
\end{tabular}

Tabla 2.

Estadísticos descriptivos para la actividad sedentaria y la actividad física realizada entre semana según género. Tiempo expresado en minutos/día (M y DT)

\begin{tabular}{|c|c|c|c|c|c|c|c|c|c|}
\hline \multirow[b]{2}{*}{ Segmentos horarios } & & \multicolumn{2}{|c|}{ Actividad Sedentaria } & \multicolumn{2}{|c|}{ Actividad Ligera } & \multicolumn{2}{|c|}{ Actividad Moderada } & \multicolumn{2}{|c|}{ Actividad Vigorosa } \\
\hline & & Chicos & Chicas & Chicos & Chicas & Chicos & Chicas & Chicos & Chicas \\
\hline $\begin{array}{l}\text { Precolegio } \\
n_{\text {chicos }}=70 \\
n_{\text {chicas }}=94\end{array}$ & $\begin{array}{c}M \\
(D T)\end{array}$ & $\begin{array}{c}\mathbf{2 3 , 5 1} \\
(10,30)\end{array}$ & $\begin{array}{c}\mathbf{2 5 , 5 1} \\
(10,15)\end{array}$ & $\begin{array}{l}23,70 \\
(8,88)\end{array}$ & $\begin{array}{l}23,50 \\
(8,13)\end{array}$ & $\begin{array}{c}5,30 \\
(3,24)\end{array}$ & $\begin{array}{c}5,11 \\
(3,86)\end{array}$ & $\begin{array}{c}2,27 \\
(3,16)\end{array}$ & $\begin{array}{c}\mathbf{1 , 2 9} \\
(1,81)\end{array}$ \\
\hline $\begin{array}{c}\text { Colegio } \\
\mathrm{n}_{\text {chicos }}=79 \\
\mathrm{n}_{\text {chicas }}=102\end{array}$ & $\begin{array}{c}M \\
(D T)\end{array}$ & $\begin{array}{l}132,43 \\
(27,43)\end{array}$ & $\begin{array}{l}124,21 \\
(26,96)\end{array}$ & $\begin{array}{c}89,43 \\
(19,93)\end{array}$ & $\begin{array}{c}87,05 \\
(17,67)\end{array}$ & $\begin{array}{l}10,61 \\
(5,33)\end{array}$ & $\begin{array}{c}9,36 \\
(6,18)\end{array}$ & $\begin{array}{c}3,13 \\
(3,28)\end{array}$ & $\begin{array}{c}\mathbf{1 , 2 5} \\
(2,15)\end{array}$ \\
\hline $\begin{array}{c}\text { Comida } \\
\mathrm{n}_{\text {chicos }}=75 \\
\mathrm{n}_{\text {chicas }}=100\end{array}$ & $\begin{array}{c}M \\
(D T)\end{array}$ & $\begin{array}{l}27,63 \\
(7,40)\end{array}$ & $\begin{array}{l}27,61 \\
(7,29)\end{array}$ & $\begin{array}{l}23,44 \\
(7,19)\end{array}$ & $\begin{array}{l}24,31 \\
(6,03)\end{array}$ & $\begin{array}{c}4,37 \\
(3,52)\end{array}$ & $\begin{array}{c}5,07 \\
(3,87)\end{array}$ & $\begin{array}{c}\mathbf{1 , 9 5} \\
(2,52)\end{array}$ & $\begin{array}{c}\mathbf{1 , 2 5} \\
(2,11)\end{array}$ \\
\hline $\begin{array}{c}\text { Tarde } 1 \\
\mathrm{n}_{\text {chicos }}=73 \\
\mathrm{n}_{\text {chicas }}=98\end{array}$ & $\begin{array}{c}M \\
(D T)\end{array}$ & $\begin{array}{c}38,62 \\
(14,72)\end{array}$ & $\begin{array}{c}39,51 \\
(12,55)\end{array}$ & $\begin{array}{c}36,16 \\
(14,93)\end{array}$ & $\begin{array}{c}34,45 \\
(11,39)\end{array}$ & $\begin{array}{c}6,22 \\
(6,08)\end{array}$ & $\begin{array}{c}5,63 \\
(4,93)\end{array}$ & $\begin{array}{c}5,26 \\
(7,60)\end{array}$ & $\begin{array}{c}\mathbf{1 , 9 2} \\
(3,06)\end{array}$ \\
\hline $\begin{array}{c}\text { Tarde } 2 \\
\mathrm{n}_{\text {chicos }}=67 \\
\mathrm{n}_{\text {chicas }}=99\end{array}$ & $\begin{array}{c}M \\
(D T)\end{array}$ & $\begin{array}{c}\mathbf{4 0 , 4 0} \\
(13,06)\end{array}$ & $\begin{array}{c}\mathbf{4 5 , 5 6} \\
(12,32)\end{array}$ & $\begin{array}{c}40,37 \\
(14,00)\end{array}$ & $\begin{array}{c}39,24 \\
(11,99)\end{array}$ & $\begin{array}{c}6,10 \\
(6,67)\end{array}$ & $\begin{array}{c}6,05 \\
(6,49)\end{array}$ & $\begin{array}{c}\mathbf{4 , 4 2} \\
(5,81)\end{array}$ & $\begin{array}{c}\mathbf{1 , 7 5} \\
(3,12)\end{array}$ \\
\hline $\begin{array}{c}\text { Noche } \\
\mathrm{n}_{\text {chicos }}=61 \\
n_{\text {chicas }}=91\end{array}$ & $\begin{array}{c}M \\
(D T)\end{array}$ & $\begin{array}{c}33,28 \\
(16,52)\end{array}$ & $\begin{array}{c}34,85 \\
(13,93)\end{array}$ & $\begin{array}{c}27,16 \\
(11,71)\end{array}$ & $\begin{array}{c}26,68 \\
(10,72)\end{array}$ & $\begin{array}{c}1,98 \\
(2,69)\end{array}$ & $\begin{array}{c}1,35 \\
(2,46)\end{array}$ & $\begin{array}{c}1,11 \\
(3,40)\end{array}$ & $\begin{array}{c}0,43 \\
(1,17)\end{array}$ \\
\hline
\end{tabular}

Nota: Se muestran en letra negrita las medias que presentan diferencias estadísticamente significativas.

Estadísticos descriptivos para la actividad sedentaria y la actividad física realizada durante el fin de semana según género. Tiempo expresado en minutos/día $(M$ y $D T)$

\begin{tabular}{|c|c|c|c|c|c|c|c|c|c|}
\hline \multirow[b]{2}{*}{ Segmentos horarios } & & \multicolumn{2}{|c|}{ Actividad Sedentaria } & \multicolumn{2}{|c|}{ Actividad Ligera } & \multicolumn{2}{|c|}{ Actividad Moderada } & \multicolumn{2}{|c|}{ Actividad Vigorosa } \\
\hline & & Chicos & Chicas & Chicos & Chicas & Chicos & Chicas & Chicos & Chicas \\
\hline $\begin{array}{c}\text { Mañana } \\
\mathrm{n}_{\text {chicos }}=68 \\
n_{\text {chicas }}=103\end{array}$ & $\begin{array}{c}M \\
(D T)\end{array}$ & $\begin{array}{c}74,54 \\
(37,84)\end{array}$ & $\begin{array}{c}71,30 \\
(47,79)\end{array}$ & $\begin{array}{c}75,07 \\
(42,26)\end{array}$ & $\begin{array}{c}68,96 \\
(36,13)\end{array}$ & $\begin{array}{c}9,16 \\
(16,25)\end{array}$ & $\begin{array}{c}6,94 \\
(8,73)\end{array}$ & $\begin{array}{c}3,01 \\
(6,12)\end{array}$ & $\begin{array}{c}1,49 \\
(3,62)\end{array}$ \\
\hline $\begin{array}{c}\text { Comida } \\
\mathrm{n}_{\text {chicos }}=70 \\
\mathrm{n}_{\text {chicas }}=104\end{array}$ & $\begin{array}{c}M \\
(D T)\end{array}$ & $\begin{array}{c}28,61 \\
(11,32)\end{array}$ & $\begin{array}{c}27,89 \\
(12,16)\end{array}$ & $\begin{array}{c}26,14 \\
(12,94)\end{array}$ & $\begin{array}{c}21,76 \\
(10,89)\end{array}$ & $\begin{array}{c}1,68 \\
(2,44)\end{array}$ & $\begin{array}{c}1,37 \\
(3,57)\end{array}$ & $\begin{array}{c}0,97 \\
(2,81)\end{array}$ & $\begin{array}{c}0,41 \\
(2,18)\end{array}$ \\
\hline $\begin{array}{c}\text { Tarde } 1 \\
\mathrm{n}_{\text {chicos }}=69 \\
\mathrm{n}_{\text {chicas }}=104\end{array}$ & $\begin{array}{c}M \\
(D T)\end{array}$ & $\begin{array}{c}44,94 \\
(20,01)\end{array}$ & $\begin{array}{c}43,24 \\
(16,33)\end{array}$ & $\begin{array}{c}40,75 \\
(19,00)\end{array}$ & $\begin{array}{c}35,56 \\
(13,86)\end{array}$ & $\begin{array}{c}5,46 \\
(9,12)\end{array}$ & $\begin{array}{c}3,59 \\
(4,99)\end{array}$ & $\begin{array}{c}3,29 \\
(7,15)\end{array}$ & $\begin{array}{c}\mathbf{0 , 8 7} \\
(1,97)\end{array}$ \\
\hline $\begin{array}{c}\text { Tarde } 2 \\
\mathrm{n}_{\text {chicos }}=70 \\
\mathrm{n}_{\text {chicas }}=103\end{array}$ & $\begin{array}{c}M \\
(D T)\end{array}$ & $\begin{array}{c}45,93 \\
(17,65)\end{array}$ & $\begin{array}{c}44,06 \\
(18,63)\end{array}$ & $\begin{array}{c}40,16 \\
(18,65)\end{array}$ & $\begin{array}{c}37,77 \\
(17,72)\end{array}$ & $\begin{array}{c}4,53 \\
(6,33)\end{array}$ & $\begin{array}{c}4,72 \\
(6,27)\end{array}$ & $\begin{array}{c}1,68 \\
(3,26)\end{array}$ & $\begin{array}{c}1,22 \\
(2,72)\end{array}$ \\
\hline $\begin{array}{c}\text { Noche } \\
\mathrm{n}_{\text {chicos }}=71 \\
\mathrm{n}_{\text {chicas }}=103\end{array}$ & $\begin{array}{c}M \\
(D T)\end{array}$ & $\begin{array}{c}34,39 \\
(20,71)\end{array}$ & $\begin{array}{c}36,21 \\
(19,93)\end{array}$ & $\begin{array}{c}33,10 \\
(26,27)\end{array}$ & $\begin{array}{c}31,31 \\
(17,80)\end{array}$ & $\begin{array}{c}4,25 \\
(7,51)\end{array}$ & $\begin{array}{c}2,53 \\
(4,53)\end{array}$ & $\begin{array}{c}1,04 \\
(1,87)\end{array}$ & $\begin{array}{c}0,66 \\
(2,43)\end{array}$ \\
\hline $\begin{array}{c}\text { Madrugada sábado } \\
\mathrm{n}_{\text {chicos }}=23 \\
\mathrm{n}_{\text {chicas }}=40\end{array}$ & $\begin{array}{c}M \\
(D T)\end{array}$ & $\begin{array}{c}17,17 \\
(20,26)\end{array}$ & $\begin{array}{c}27,37 \\
(39,72)\end{array}$ & $\begin{array}{c}11,95 \\
(12,38)\end{array}$ & $\begin{array}{c}27,27 \\
(47,74)\end{array}$ & $\begin{array}{c}0,60 \\
(1,27)\end{array}$ & $\begin{array}{c}2,12 \\
(5,77)\end{array}$ & $\begin{array}{c}0,26 \\
(0,91)\end{array}$ & $\begin{array}{c}0,17 \\
(0,59)\end{array}$ \\
\hline $\begin{aligned} \text { Madrugada domingo } \\
\mathrm{n}_{\text {chicos }}=39 \\
n_{\text {chicas }}=50\end{aligned}$ & $\begin{array}{c}M \\
(D T)\end{array}$ & $\begin{array}{c}31,36 \\
(38,90)\end{array}$ & $\begin{array}{c}22,54 \\
(32,92)\end{array}$ & $\begin{array}{c}30,95 \\
(39,69)\end{array}$ & $\begin{array}{c}20,24 \\
(28,61)\end{array}$ & $\begin{array}{c}3,67 \\
(10,79)\end{array}$ & $\begin{array}{c}1,46 \\
(4,62)\end{array}$ & $\begin{array}{c}0,74 \\
(2,02)\end{array}$ & $\begin{array}{c}0,22 \\
(0,91)\end{array}$ \\
\hline
\end{tabular}

Nota: Se muestran en letra negrita las medias que presentan diferencias estadísticamente significativas.

actividad vigorosa que las chicas (2,27 $\pm 3,16$ vs. $1,29 \pm 1,81 ; p<.05$, $\left.\eta^{2}=.037\right)$. Los chicos mostraron mayor AF vigorosa que las chicas en los segmentos horarios ‘Colegio' (3,13 $\pm 3,28$ vs. $1,25 \pm 2,15 ; p<.001$, $\left.\eta^{2}=.110\right)$, 'Comida' ( $1,95 \pm 2,52$ vs. $\left.1,25 \pm 2,11 ; p<.05, \eta^{2}=.024\right)$ y 'Tarde 1' (5,26 $\pm 7,60$ vs. $\left.1,92 \pm 3,06 ; p<.001, \eta^{2}=.077\right)$. En el segmento horario 'Tarde 2', las chicas mostraron mayor actividad sedentaria que los chicos (45,56 $\pm 12,32$ vs. $40,40 \pm 13,06 ; p<.01, \eta^{2}=.048$ ), mientras que los chicos mostraron mayor actividad vigorosa que las chicas $\left(4,42 \pm 5,81\right.$ vs. $\left.1,75 \pm 3,12 ; p<.001, \eta^{2}=.091\right)$. No se encontraron diferencias estadísticamente significativas en el segmento horario
'Noche'.

Respecto al fin de semana, los resultados también mostraron diferencias estadísticamente significativas $(p<.05)$ según género (Tabla 1$)$. En la Tabla 3, se indica la $M$ y la $D T$ de los minutos en actividad sedentaria y en las diferentes intensidades de práctica de $\mathrm{AF}$ durante el fin de semana. Concretamente, en el segmento horario 'Tarde 1' del fin de semana, los chicos mostraron mayor actividad vigorosa que las chicas (3,29 $\pm 7,15$ vs. $0,87 \pm 1,97 ; p<.01$, $\left.\eta^{2}=.044\right)$. No existieron diferencias estadísticamente significativas en el resto de segmentos horarios correspondientes al fin de semana.

Por último, debe destacarse que las diferencias estadísticamente significativas que presentaron mayor tamaño del efecto fueron las encontradas para actividad vigorosa a favor de los chicos en tres segmentos horarios entre semana (por orden, ‘Colegio', 'Tarde 2'y ‘ Tarde 1 ').

\section{Discusión}

El objetivo de este estudio fue analizar las diferencias según género en el tiempo empleado por los adolescentes en actividad sedentaria y AF ligera, moderada y vigorosa en diferentes segmentos horarios del día, tanto entre semana como en fin de semana. Atendiendo al segmento horario ‘Precolegio', la mayor participación de los chicos en AF vigorosa y la mayor participación de las chicas en actividad sedentaria presenta cierta relación con los resultados de Aibar et al. (2014), que indicaron mayor nivel deAF moderada-vigorosa por parte de los chicos durante el 'tiempo de transporte al colegio’. Sin embargo, el estudio de Bailey et al. (2012) encontró que las chicas mostraban mayor tiempo en AF ligera que los chicos en el segmento horario correspondiente al transporte al centro escolar. Los estudios sobre el uso de transporte activo para asistir a la escuela no sirven para despejar esta controversia, ya que hay estudios que indican que los chicos son más activos que las chicas en su trayecto a la escuela (Chillón et al., 2011), mientras que otros indican lo contrario (Chillón et al., 2009). En cualquier caso, la promoción del transporte activo para asistir a la escuela representa una alternativa interesante para la promoción de un estilo de vida activo. Sería aconsejable que la administración pública, los profesionales de la salud y los responsables educativos colaboraran para que los adolescentes fueran al colegio andando o en bicicleta, o mediante otros tipos de transporte activo, y pudieran hacer el trayecto por entornos seguros y agradables (Chillón, 2011). A la vista de nuestros resultados, las chicas deberían ser la principal población diana de estas estrategias. Futuros estudios podrían analizar si las diferencias en el segmento horario ‘Precolegio’ se deben a posibles diferencias en el tipo de transporte a la escuela o en la práctica de actividad físicodeportiva a primera hora de la mañana, antes de asistir a la escuela, o antes de comenzar las clases, en las propias instalaciones escolares.

En el segmento horario ‘Colegio', los chicos mostraron mayor AF vigorosa que las chicas, siendo esta diferencia la que presentó mayor tamaño del efecto en este estudio. Este resultado concuerda con los 
datos hallados en otras investigaciones. Concretamente, Aibar et al. (2014) observaron que el ‘tiempo en la escuela’ era uno de los segmentos horarios donde mayores diferencias de AF moderada/vigorosa existían a favor de los chicos. Además, Bailey et al. (2012), al dividir el horario escolar en varios segmentos horarios, hallaron que los chicos mostraban mayores niveles de AF vigorosa durante el recreo de la mañana, y de AF moderada y vigorosa durante el descanso para el almuerzo (lunch). La menor participación en AF de las chicas durante el horario escolar representa un dato relevante, cuando cursan junto a los chicos Educación Física y supuestamente disponen de los mismos horarios, instalaciones, recursos y oferta de actividades escolares.

Para aumentar los niveles de AF de las chicas durante el horario escolar podrían dinamizarse los recreos, para que fuesen más activos con actividades que se ajustaran mejor a las preferencias de las chicas. Se trataría de evitar que los chicos dominaran el espacio disponible para el juego con actividades más competitivas como el fútbol, promocionando en los recreos actividades físico-deportivas que impliquen comunicación y relación social, y tareas como saltar o bailar (Blatchford, Baines, y Pellegrini, 2003; Boyle, Marshall, y Robeson, 2003). Para lograr estas transformaciones, los recreos deberían formar parte de programas globales de promoción de la AF en los centros escolares, y sería necesario que los centros facilitaran el acceso de los alumnos al material e instalaciones deportivas de la escuela (Ridgers, Salmon, Parrish, Stanley, y Okely, 2012).

El profesorado de Educación Física también puede desempeñar una labor fundamental para aumentar la participación en AF de las chicas, incorporando en sus clases contenidos que se ajusten más a sus características, necesidades y preferencias. Lamentablemente, en algunas ocasiones, la Educación Física aún sigue siendo un ámbito ligado a la masculinidad en el que algunas chicas se perciben menos valoradas y con menos oportunidades de participación (Beltrán-Carrillo, DevísDevís, Peiró-Velert, y Brown, 2012). Desde esta perspectiva, sería interesante obtener información, a principio de curso, sobre las preferencias de AF y deporte de chicas y chicos, mediante cuestionarios, entrevistas o grupos de discusión, de modo que el currículum pudiera estar equiparado en función de las preferencias de los alumnos de ambos géneros. Por ejemplo, en un estudio reciente en Educación Física(Murillo, Julián, García-González, Abarca-Sos, y Zaragoza, 2014), se puso de manifiesto que la unidad didáctica de salto con combas era la única en la quelas chicas mostraban una mayor puntuación en competencia percibida que los chicos, lo que podría explicar la inexistencia de diferencias estadísticamente significativas según género en los niveles deAF moderada-vigorosa durante la impartición de dicho contenido. Sin embargo, en contenidos como el atletismo, voleibol y baloncesto, los niveles de AF moderada-vigorosa durante las clases fueron significativamente superiores en los chicos.

Respecto al segmento horario 'Colegio', futuros estudios podrían analizar las diferencias en actividad sedentaria y AF ligera, moderada y vigorosa en distintos momentos del horario escolar, diferenciando entre clases de Educación Física, recreos y clases en el aula. Se trataría de un planteamiento semejante al del estudio de Bailey et al. (2012), pero adaptando los segmentos horarios al contexto español y diferenciando las clases de Educación Física de las clases de aula.

En el segmento horario ‘Comida' entre semana los chicos presentaron mayor actividad vigorosa que las chicas. En línea con este resultado, el estudio de Bailey et al. (2012) encontró que los chicos mostraban mayor actividad moderada y vigorosa durante el descanso escolar para el almuerzo (lunch). Sin embargo, el segmento horario equivalente en Reino Unido al de la comida en España presenta claras diferencias culturales. En el caso de nuestro estudio, este segmento horario representa normalmente el periodo en que los adolescentes van del centro educativo a casa, el periodo de la comida y los momentos previos y posteriores a ella. Sería interesante que futuros estudios siguieran indagando en los patrones de AF durante este segmento horario, para saber si las posibles diferencias se deben a diferencias en el tipo de transporte del colegio a casa u a otros factores.

Los chicos mostraron niveles más elevados de actividad vigorosa en los segmentos horarios 'Tarde 1'y 'Tarde 2' entre semana. Estas diferencias son las que presentaron mayor tamaño del efecto, tras las encontradas en AF vigorosa en el segmento horario 'Colegio', y podrían deberse a una mayor participación por parte de los chicos en actividades extraescolares en horario de tarde. Además, algunos autores han encontrado una mayor práctica de $\mathrm{AF}$ vigorosa por parte de los chicos durante programas de AF llevados a cabo por las tardes después del horario escolar (Trost, Rosencranz, y Dzewaltowski, 2008). Desde una perspectiva de salud pública, podría ser interesante ofertar programas de promoción de AF y salud por las tardes entre semana, dirigidos especialmente a atender las preferencias de las chicas. Para el diseño y aplicación de estos programas, debería tenerse en cuenta que las chicas valoran especialmente la existencia de un clima social inclusivo, donde haya una elevada presencia de juegos cooperativos y actividades en grupo, de modo que puedan aflorar relaciones sociales positivas (Zarrett, Sorensen, y Skiles, 2015).

En el segmento horario 'Tarde 2' entre semana, las chicas mostraron mayor actividad sedentaria que los chicos. Debe considerarse que las mejoras en la salud no solo están relacionadas con mayor participación en AF moderada-vigorosa, sino también con una menor participación en actividad sedentaria (Ramos et al., 2012). Por lo tanto, la promoción de la AF ligera también debe formar parte de los planes de promoción de estilos de vida activos en niños y adolescentes (Arto, 2015). Sería interesante que durante este segmento horario las chicas trataran de reducir el tiempo en actividades sedentarias incorporando el transporte activo en las actividades de la vida diaria (pasear, bicicleta, subir y bajar escaleras, etc.) o haciendo pequeños descansos activos entre las horas de estudio que impliquen caminar, movimientos articulares y ejercicios de flexibilidad. Asimismo, pasear con familiares y amigos/as podría ser una alternativa interesante para ocupar el tiempo de ocio de estos segmentos horarios.

Las diferencias en los patrones de AF encontradas en este estudio durante las tardes entre semana contrastan con los resultados de Bailey et al. (2012), que no encontraron diferencias estadísticamente significativas en actividad sedentaria o en AF ligera, moderada, vigorosa 'después del colegio’. Es probable que las diferencias en horarios escolares, clima, horas de luz, comidas y costumbres, entre Reino Unido y España, afecten a la diversidad en los patrones de AF de los adolescentes. Este dato alerta de la necesidad de ajustar las estrategias de promoción de la $\mathrm{AF}$ a los diferentes contextos socioculturales y ambientales de las poblaciones diana.

En lo que concierne al fin de semana, llama la atención la homogeneidad en los patrones de AF que presentan los chicos y chicas de este estudio, en comparación con las diferencias halladas entre semana. Sólo se encontró una diferencia estadísticamente significativa, debida a una mayor participación en $\mathrm{AF}$ vigorosa de los chicos en el segmento horario 'Tarde 1' del fin de semana. Esta diferencia podría deberse a una mayor participación de los chicos en deportes como el fútbol, el fútbolsala o el baloncesto (Chillón, Tercedor, Delgado, y González-Gross, 2002), que suelen requerir la participación en competiciones los fines de semana. Sería deseable la implicación directa de padres y madres en la promoción de la AF vigorosa de las chicas durante los fines de semana. Las familias podrían entender el ocio activo como una oportunidad para pasar un buen rato en familia los fines de semana, teniendo en cuenta que la coincidencia en el tiempo de ocio de los distintos miembros de la unidad familiar probablemente sea menor entre semana.

Por último, debe destacarse la ausencia de diferencias estadísticamente significativas en los patrones de AF de los adolescentes de este estudio durante los segmentos horarios de las noches y las madrugadas. Estos datos, unidos a que las diferencias en $\mathrm{AF}$ encontradas en los segmentos horarios 'Tarde 1 entre semana (aún de día)' y 'Tarde 2' entre semana (ya había anochecido)' son semejantes, nos llevan a descartar una posible hipótesis de que las chicas de este estudio realicen menor AF por enfrentarse a más barreras que los chicos para salir de casa e implicarse en diferentes actividades cuando ya ha anochecido. Esta hipótesis se contemplaba, en un principio, debido a que algunos factores socio-ambientales como la inseguridad ciudadana están 
relacionados con menor participación en AF por parte de los adolescentes (Ferreira et al., 2007). Además, estudios previos en el contexto español identifican como barreras para la práctica de AF en adolescentes ‘no me dejan salir solo’ o ‘no es seguro jugar en la calle’ (Serra-Puyal, Generelo-Lanaspa, y Zaragoza-Casterad, 2010), y han mostrado que las chicas dependen más de sus progenitores para el transporte hacia los lugares de práctica física, por el miedo y la preocupación que genera en las familias el hecho de que vayan solas por determinados lugares de la ciudad cuando ya ha anochecido (Devís-Devís, Beltrán-Carrillo, y PeiróVelert, 2015). Probablemente las cuestiones relativas a la inseguridad ciudadana sólo sean una barrera en grandes ciudades y zonas con elevados índices de criminalidad (Devís-Devís et al., 2015).

La ausencia de diferencias estadísticamente significativas en los segmentos horarios correspondientes a la noche y las madrugadas del fin de semana nos lleva a descartar igualmente la hipótesis de que las chicas realicen mayor $\mathrm{AF}$ moderada o vigorosa que los chicos, durante su ocio nocturno del fin de semana, por su mayor preferencia por actividades relacionadas con el baile (Chillón et al., 2002) y menor preferencia por el uso de medios tecnológicos como los videojuegos convencionales y la televisión (Beltrán-Carrillo, Beltrán-Carrillo, González-Cutre, Biddle, y Montero-Carretero, 2015; Valencia-Peris, Devís-Devís, y Peiró-Velert, 2014).

Diversas limitaciones de este estudio deben ser reconocidas. En primer lugar, los datos procedentes de los acelerómetros fueron almacenados en epochs de 60 segundos. Epochs más reducidos hubieran ofrecido medidas más rigurosas para actividad sedentaria y para AF ligera, moderada y vigorosa. A lo largo del tiempo, los estudios han utilizado epochs de 60, 15 y 5 segundos, pero la literatura reciente recomienda el uso de epochs de 1 segundo (Calahorro-Cañada et al., 2015). Otra limitación de este estudio fue que el modelo de acelerómetro utilizado no permitió medir el tiempo dedicado a la AF en el medio acuático. Pese a las limitaciones mencionadas, el uso del acelerómetro para la medida de la AF debe destacarse como un punto fuerte de este estudio, según las recomendaciones de la literatura para este tipo de investigaciones (Aibar et al., 2014; Bailey et al., 2012). También debe destacarse el riguroso y laborioso proceso de reducción y análisis de datos, que arroja luz sobre las diferencias en los patrones de AF de chicos y chicas en muchos segmentos horarios del día, tanto entre semana como en fin de semana, varios de ellos inexplorados previamente. Además, este estudio presenta una discusión de los resultados, y una serie de recomendaciones para la promoción de la AF, que pueden ser de interés para la comunidad de investigadores y profesionales preocupados por la promoción de la AF y la salud de los adolescentes.

\section{Agradecimientos}

Los datos de este estudio proceden de un proyecto de investigación financiado por la Universidad Miguel Hernández-Bancaja (diligencia de publicación del 22/09/2011, n 11859 ) y por la Conselleria d’Educació, Cultura i Esport de la Generalitat Valenciana (GV/2013/101)

\section{Referencias}

Aibar,A., Bois, J.E., Zaragoza, J., Generelo, E., Paillard, T., y Fairclough, S. (2014).Weekday and weekend physical activity patterns of French and Spanish adolescents. European Journal of Sport Science, 14(5), 500-509. doi: 10.1080/ 17461391.2013.829127

Arto, G. (2015). Children's segment specific light physical activity across two years of school-based program. Journal of Physical Education and Sport, 15(1), 88-95. doi: 10.7752/jpes.2015.01015

Bailey, D. P., Fairclough, S. J., Savory, L.A., Denton, S. J., Pang, D., Deane, C. S., y Kerr, C. J. (2012). Accelerometry-assessed sedentary behaviour and physical activity levels during the segmented school day in 10-14-years-old children: The HAPPY study. European Journal of Pediatrics, 171(12), 1805-1813. doi: 10.1007/s00431-012$1827-0$

Beltrán-Carrillo, V. J., Beltrán-Carrillo, J. I., González-Cutre, D., Biddle, S. J. H., y MonteroCarretero, C. (2015). Are active videogames associated with less screen media or conventional physical activity? Games and Culture. Publicación en línea avanzada. doi: 10.1177/1555412015574941

Beltrán-Carrillo, V. J., Devís-Devís, J., Peiró-Velert, C., y Brown, D. H. K. (2012). When physical activity participation promotes inactivity: Negative experiences of Spanish adolescents in Physical Education and sport. Youth and Society, 44(1), 3-27. doi: 10.1177/0044118X10388262

Blatchford, P., Baines, E., y Pellegrini,A. D. (2003). The social context of school playground games: Sex and ethnic difference, and changes over time after entry to junior school.
British Journal of Developmental Psychology, 21, 481-505. doi: 10.1348/ 026151003322535183

Boyle, E., Marshall, N. L., y Robeson, W. W. (2003). Gender at play: Fourth-grade girls and boys on the playground. American Behavioral Scientist, 46, 1326-1345. doi: $10.1177 / 0002764203046010004$

Calahorro-Cañada, F, Torres-Luque, G, López-Fernández I Santos-Lozano, A. Garatachea, N., y Álvarez-Carnero, E. (2015). Actividad física y acelerometría; orientaciones metodológicas, recomendaciones y patrones. Nutrición Hospitalaria, 31(1), 115128. doi: 10.3305/nh.2015.31.1.7450

Catellier, D. J., Hannan, P. J., Murray, D. M., Addy, C. L., Conway, T. L., Yang, S., y Rice, J. C. (2005). Imputation of missing data when measuring physical activity by accelerometry. Medicine and Science in Sports and Exercise, 37, S555-S562. doi:10.1249/01.mss.0000185651.59486.4e

Chillón, P., Ortega, F. B., Ruiz, I. D., Bourdeaudhuij, I. D., Martínez-Gómez, D., VicenteRodriguez, G, ... Sjöström, M. (2011). Active commuting and physical activity in adolescents from Europe: Results from the HELENA study. Pediatric Exercise Science, 23(2), 207-217.

Chillón, P., Ortega, F. B., Ruiz, J. R., Pérez, I. J., Martín-Matillas, M., Valtueña, J., ... Delgado, M. (2009). Socio-economic factors and active commuting to school in urban Spanish adolescents: the AVENA study. European Journal of Public Health, 19(5), 470476. doi:10.1093/eurpub/ckp048

Chillón, P., Tercedor, P., Delgado, M., y González-Gross, M. (2002). Actividad físicodeportiva en escolares adolescentes. Retos: Nuevas tendencias en Educación Física, Deporte y Recreación, 1, 5-12.

Devís-Devís, J., Beltrán-Carrillo, V. J., y Peiró-Velert, C. (2015). Exploring socio-ecological factors influencing active and inactive Spanish students in years 12 and 13. Sport, Education and Society, 20(3), 361-380. doi: 10.1080/13573322.2012.754753

Evenson, K. R., Cattellier, D., Gill, K., Ondrak, K., y McMurray, R. G (2008). Calibration of two objective measures of physical activity for children. Journal of Sports Sciences, 26, 1557-1565. doi: 10.1080/02640410802334196

Ferreira, I., van der Horst, K., Wendel-Vos, W., Kremers, S., van Lenthe, F. J., y Brug, J. (2007). Environmental correlates of physical activity in youth - A review and update. Obesity Reviews, 8, 129-154. doi: 10.1111/j.1467-789X.2006.00264.x

Guthold, R., Cowan, M. J., Autenrieth, C. S., Kann, L. y Riley, L. M. (2010). Physical activity and sedentary behavior among schoolchildren: A 34 Country comparison. The Journal of Pediatrics, 157(1), 43-49. doi: 10.1016/j.jpeds.2010.01.019

Laguna, M., Ruiz, J., Gallardo, C., García-Pastor, T., Lara, M. T., y Aznar (2013). Obesity and physical activity patterns in children and adolescents. Journal of Paediatrics and Child Health, 49, 942-949. doi: 10.1111/jpc.12442

Murillo, B., Julián, J. A., García-González, L., Albarca-Sos, A., y Zaragoza, J. (2014). Influencia del género y de los contenidos sobre la actividad física y la percepción de competencia en Educación Física. RICYDE. Revista Internacional de Ciencias del Deporte, 10(36), 131-143. doi: 10.5232/ricyde2014.03604

Peiró-Velert, C., Valenciano-Valcárcel, J., Beltrán-Carrillo, V. J., y Devís-Devís, J. (2014). Variabilidad de la actividad física en adolescentes españoles de 17-18 años en función del tipo de jornada y época del año. Revista de Psicología del Deporte, 23(2), 347-354.

Ramos, P., Rivera, F., Moreno, C., y Jiménez-Iglesias, A. (2012). Análisis de clúster de la actividad física y las conductas sedentarias de los adolescentes españoles, correlación con la salud biopsicosocial. Revista de Psicologia del Deporte, 21(1), 99106.

Ridgers, N. D., Salmon, J., Parrish, A., Stanley, R. M., y Okely, A. D. (2012). Physical activity during school recess: A systematic review. American Journal of Preventive Medicine, 43(3), 320-328. doi: 10.1016/j.amepre.2012.05.019

Santos-Lozano, A., Marin, P. J., Torres-Luque, G, Ruiz, J. R., Lucia, A., y Garatachea, N. (2012). Technical variability of the GT3X accelerometer. Medical Engineering of Physics, 34(6), 787-790. doi: 10.1016/j.medengphy.2012.02.005

Serra-Puyal, J. R., Generelo-Lanaspa, E., y Zaragoza-Casterad, J. (2010). Barreras para la realización de actividad física en adolescentes en la provincia de Huesca. Revista Internacional de Medicina y Ciencias de la Actividad Física y el Deporte, 10(39), 470-483.

Telama, R., Yang, X., Leskinen, E., Kankaanpää, A., Hirvensalo, M., Tammelin, T., . . Raitakari, O. T. (2014). Tracking of physical activity from early childhood through youth into adulthood. Medicine \& Science in Sports \& Exercise, 46, 955-962. doi: 10.1249/ MSS.0000000000000181.

Trost, S. G, Loprinzi, P. D., Moore, R., y Pfeifer, K.A. (2011). Comparison of accelerometer cut points for predicting activity intensity in youth. Medicine \& Science in Sports \& Exercise, 43, 1360-1368. doi: 10.1249/MSS.0b013e318206476e

Trost, S. G., Rosenkranz, R. R., y Dzewaltowski, D. (2008). Physical activity levels among children attending after-school programs. Medicine \& Science in Sports \& Exercise, 40(4), 622-629, doi: 10.1249/MSS.0b013e318161eaa5

Valencia-Peris, A., Devís-Devís, J., y Peiró-Velert, C. (2014). El uso sedentario de medios tecnológicos de pantalla: perfil sociodemográfico de los adolescentes españoles. Retos: Nuevas tendencias en Educación Física, Deporte y Recreación, 26, 21-26.

Welk, G. J., Eisenmann, J. C., y Dollman, J. (2006). Health-related physical activity in children and adolescents: a bio-behavioral perspective. En D. Kirk, M. O’Sullivan, y D. Macdonald (Eds.), The Handbook of Physical Education (pp. 666-684). London: Sage. doi: 10.4135/9781848608009

World Health Organization. (2011). Global status report on noncommunicable diseases 2010. Geneva, Switzerland: WHO Press. Recuperado de http://www.who.int/nmh/ publications/ncd_report_full_en.pdf

Yan, A. F., Voorhees, C. C., Beck, K. H., yWang, M. Q. (2014). A social ecological assessment of physical activity among urban adolescents. American Journal of Health Behavior, 38(3), 379-391. doi: 10.5993/AJHB.38.3.7

Zarrett, N., Sorensen, C., y Skiles, B. (2015). Physical and social-motivational contextua correlates of youth physical activity in under-resourced afterschool programs. Health Education \& Behavior, 1, 1-12. doi: 10.1177/1090198114564502 